\title{
Overgrown beak in a budgerigar (Melopsittacus undulatus)
}

Raffaele Melidone, Dr. Med. Vet. \& Jörg Mayer, Dr. Med. Vet., MSc

A three-year-old female budgerigar (Melopsittacus undulatus) was presented to a private practice, Centro Veterinario Trinacria, in Palermo, Italy for chronic, severe, abnormal overgrowth of the beak (rhamphotheca and gnathotheca) (Fig. 1). 'Parakeet' is a general term for a small, long-tailed, slender-bodied parrot, in the Psittacine family. Budgerigars are a specific type of parakeet and originate from Australia. In the US, budgerigars are commonly called parakeets, but in the UK are called budgerigars or budgies.

The bird's owner had housed the parakeet in an appropriate sized cage with a male budgerigar of unknown age. The diet consisted of mixed seeds and a small variety of fresh fruits and vegetables. According to the owner, the overgrowth of the beak started 4 months before presentation. Despite the abnormal growth of the beak, the bird continued to eat and behave normally. The owner became concerned about the female budgerigar only after the male companion bird began to show similar pruritic clinical signs.

On physical examination, the budgerigar appeared to be bright, alert and responsive and was able to use its beak despite the deformity. The bird also had several crusty, pruritic lesions around the cere, periorbitally on both eyes (Fig. 1) and on both legs and feet. The bird's body condition was within normal limits and the rest of the physical examination was unremarkable.

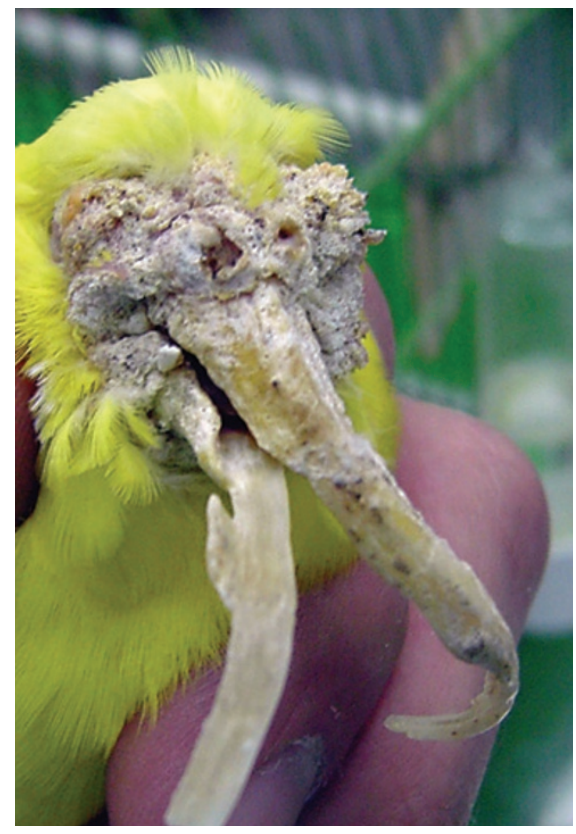

FIGURE 1 | A three-year-old female budgerigar with a 4-month duration of severe beak overgrowth and crusty, pruritic lesions around the cere and periorbitally on both eyes.

We used a sterile \#10 surgical blade dipped in Hoyer's solution (a glycerin solution) with eosin dye to scrape the main lesion on the cere and transferred the scraping from the scalpel blade to the microscope slide and applied a coverslip. Microscopic examination (at 200× magnification)

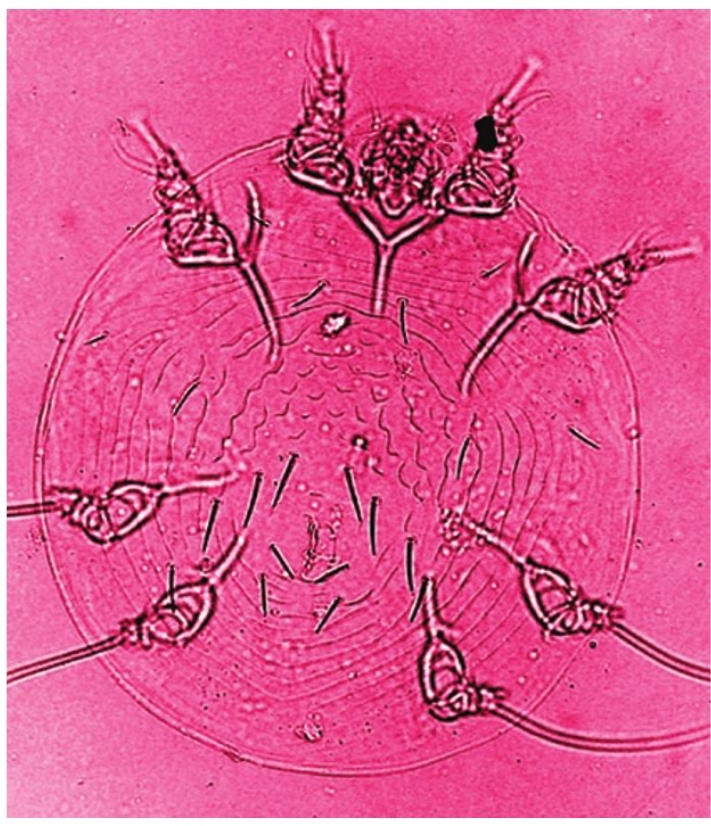

FIGURE 2 | Parasite obtained from a scraping of crusty lesion on the cere of the budgerigar in Figure 1. The parasite is preserved in eosin-stained Hoyer's solution and viewed under mediumpower magnification.

revealed live parasites (Fig. 2).

What are the parasites? How do you treat the birds for these parasites? Are the parasites the cause of the lesions?

\section{What's your diagnosis?}

